\title{
Histopathologic spectrum of adrenal lesions
}

\section{Saritha Karre', Amrutha Gorva ${ }^{2, *}$, Chandrakumar Shanmugam ${ }^{3}$, V. D. Praveen Kumar Gorrela ${ }^{4}$, Satyanarayana Veeragandham ${ }^{5}$}

${ }^{1}$ Assistant Professor, Dept. of Pathology, ESIC Medical College, Sanathanagar, Hyderabad, ${ }^{2}$ Assistant Professor, Dept. of Pathology, Mandya Institute of Medical Sciences, Mandya, Karnataka, ${ }^{3}$ Associate Professor, ${ }^{4}$ Junior Resident, Dept. of Pathology, ESIC Medical College, Sanathanagar, Hyderabad, ${ }^{5} \mathrm{HOD}$, Dept. of Pathology, Kamineni Institute of Medical Sciences, Narketpalli, Telangana, India

*Corresponding Author:

Email: amugorva@gmail.com

Received: $20^{\text {th }}$ January, 2018

Accepted: $26^{\text {th }}$ March, 2018

\begin{abstract}
Introduction: The bipartite adrenal gland has tumors arising from cortex, medulla or as metastasis from extra-adrenal sites. Distinguishing benign from malignant types is a challenging task for pathologists, this study gives an insight into adrenal lesions with their histopathological and immunohistochemical findings and its role in categorizing these tumors to aid in their diagnosis and proper management.

Aim: To study the Histopathological patterns of adrenal lesions with clinical correlation.

Materials and Methods: In a five year period10 surgical specimens of adrenal tumors were resected and studied between July 2010 to June 2015 at Kamineni Institute of Medical Sciences, Narketpally and King Koti. Tissues were processed and stained sections were studied for histopathological patterns and IHC markers used were vimentin, synaptophysin, NSE, chromogranin immunohistochemistry was done wherever necessary and the markers used were vimentin, synaptophysin, NSE, chromogranin.

Result: Out of ten adrenal lesions we have studied, two cases were adrenal cysts, ${ }^{1}$ three were cortical adenoma, one case is Adrenocortical carcinoma-positive for synaptophysin, vimentin, two cases were neuroblastoma-positive for NSE, chromogranin and synaptophysin and two cases are pheochromocytoma - positive for chromogranin.

Conclusion: Pathological evaluation and immunohistochemical profiling has got important role in categorizing adrenal tumors along with clinical, radio logical and biochemical inputs and their subsequent management.
\end{abstract}

Keywords: Adrenal cyst, Adrenal adenoma and Carcinoma (Weiss system), Neuroblastoma, Pheochromocytoma.

\section{Introduction}

A pair of adrenals are located in the retroperitoneum on the superomedial portion of both kidneys. They have structurally and functionally two distinctive zones cortex and medulla arising from mesoderm and neuroectoderm ${ }^{2}$ respectively. The primary function of cortex is to secrete cortisol, aldosterone and sex steroids and medulla is to secrete catecholamines. Tumors arising from cortex are mainly adenomas and carcinomas where as from medulla are pheochromocytomas and neuroblastomas.

Adreno cortical carcinomas are very rare tumors with a frequency of 2 in a million per year. The relative incidence among malignant tumors is $2 \%$ and shows a bimodal presentation with peak occurence at $<5$ years and 50-70 years. Hereditary syndromes like LiFraumeni, Beckwith Wiedemann and Carney complex are associated with these tumors and account only for smaller percentage of cases.

Non neoplastic lesions associated with adrenals include hypoplasia, hyperplasia, cytomegaly, cysts, nodules and accessory tissue. Most of these are nonfunctional even though some functional tumors may show clinical features like virilisation, cushingoid features, feminisation and hyperaldosteronism. ${ }^{3}$ The current study aims at studying histopathological patterns of adrenal lesions with immuno-histochemical and clinical correlation.

\section{Materials and Methods}

A five yearstudy was done at Kamineni Institute of Medical Sciences, Narketpally from July 2010 to June 2015. Clinical findings were recorded with clinical details of all subjects with respect to symptoms at presentation, age and gender. Relevant radiological investigation i.e adrenal ultrasound, intravenous pyelogram and computerized tomography scans for primary tumors and metastasis were done. The adrenocortical functions (hormonal evaluation) and provisional diagnosis were correlated with histopathological findings.

\section{Case Definition}

The criteria for cushings syndrome include clinical features like weight gain, moon face, buffalo hump, centripetal fat distribution with/without hypertension along with biochemical investigations like Dexamethasone suppression test, Serum \& urinary levels showing high cortisol with suppressed ACTH.

The affect of sex steroids can be feminising like precocious puberty - thelarche, pubarche, menarche or virilising like clitoromegaly, macrogenitosomia, hirsutism, deep voice, increased muscle mass, acne in 
females. Blood investigations show elevated $17 \mathrm{OH}$ progesterone, androgens, estrogens and decreased FSH. ${ }^{4,5}$

The typical features of pheochromocytoma ${ }^{6}$ include intermittent headaches, sweating, palpitations, unintentional weight loss and paleness due to sympathomimetic affect of catecholamines, along with increased $24 \mathrm{hr}$ Urinary catecholamine, vanillylmandelic acid and metanephrines.

The total 10 resected adrenal tumors were collected which included both non-neoplastic and neoplastic and were divided based on the nature of behavior and site of origin, grossing was done and histopathological sections were taken. Microscopic evaluation was done after proper staining. Weiss System ${ }^{7-9}$ which is considered gold standard was used to distinguish between benign and malignant lesions.

The presence of 3 or more of the following features is correlated with malignant behaviour - Weiss criteria. $^{10}$

1. High nuclear grade (Fuhrman grade system)

2. $>5$ mitotic figures/50 HPF

3. Atypical mitotic figures

4. Eosinophilic cytoplasm in $>75 \%$ of tumour cells

5. Diffuse architecture in $>1 / 3$ rd of the tumour

6. Necrosis

7. Venous invasion

8. Sinusoidal invasion

9. Capsular invasion

In relevant cases to support the working diagnosis, immunohistochemistry was performed with the markers vimentin, synaptophysin (in adrenal carcinoma); NSE, chromogranin, synaptophysin (in neuroblastoma) and chromogranin (in pheochromocytoma) and the classification of neuroblastomas were done by counting number of cells per each high power field and taking average of 10 fields (Mitotic Karyotic Index).

\section{Results}

The present study statistics show female preponderance ( 8 out of 10 cases) of adrenal lesions with a F:M ratio 4:1 and right domination (7 out of 10 cases) with right : left ratio 2.3:1. 6 out of 10 presented with mass per abdomen $(60 \%)$ and of which 5 are associated with intermittent pain abdomen. 2 out of 10 showed low grade fever $(20 \%) .1$ out of 10 with hypertension and myopathies $(10 \%)$, another 1 out of $10(10 \%)$ with features of cushings syndrome and 2 out of $10(20 \%)$ presented with giddiness and sweating.

The two adrenal cysts $(20 \%)$ were presented with intermittent pain abdomen and normal blood and urine investigations. They were reported as adrenal Pseudocyst ${ }^{11}$ and adrenal endothelial cyst. ${ }^{12}$

Adrenal adenomas were 3 in total $(30 \%)$ out of which 2 showed endocrine manifestations. One with Hypertension with elevated Aldosterone levels $(585.51 \mathrm{pg} / \mathrm{ml})$ and normal cortisol and other with elevated blood and urine cortisol and with prominent cushings features.

A single case of adrenal cortical carcinoma ${ }^{13}$ was reported $(10 \%)$ in $60 \mathrm{yr}$ old male patient, he who presented with dull pain in lumbar region and swelling in the right hypogastrium. CT images showed cystic mass admixed with peripheral solid area arising from superior pole of right kidney. Biochemical investigations of FBS \& Creatinine \& BUN were within normal range. Gross examination showed Large oval grey brown soft tissue mass with hemorrhagic and solid areas measuring $20 \times 12 \times 8.5 \mathrm{~cm}$ with ureter at one end measuring $5.5 \times 0.5 \mathrm{~cm}$. Cut section showed capsulated tumor mass with compressed kidney measuring 8.4 X 4 X $2.5 \mathrm{~cm}$. Cyst was measuring $18 \mathrm{X}$ 13 X $6 \mathrm{~cm}$. The tumor was predominantly cystic filled with mucinous material and with extensive necrosis and hemorrhagic and with solid areas at the periphery. There is no demarcation between kidney and tumor mass grossly.

Microscopy showed well encapsulated tumor with extensive areas of hemorrhage. Predominantly glandular pattern is observed with areas of diffuse papillary and trabecular pattern in between. Individual tumor cells are round to oval with vesicular nucleus, predominant nucleoli and scanty eosinophilic cytoplasm. At some areas collections of foamy macrophages and cholesterol clefts are noticed. Sections of kidney adjacent to tumor tissue shows compressed thick fibrous wall with one focus showing tumor tissue invading parenchyma of kidney in glandular pattern. Glomeruli adjacent to tumor tissue shows hyalinisation. The tumor was positive for immunohistochemistry markers vimentin, synaptophysin and negative for cytokeartin. TNM staging of this particular tumor was Stage III as it was more than $5 \mathrm{~cm}$ with kidney invasion and no lymphnode metastasis (Fig. 3).

It has fullfilled the weiss system criteria for malignant adrenocortical tumors with features of Eosinophilic cytoplasm in $>75 \%$ of tumor cells, capsular invasion and features of necrosis.

2 out of 10 were neuroblastomas, reported in children of 12 months and 18 months respectively. They presented with complaints of low grade fever and mass abdomen. One case had elevated urine VMA (vinyl mandelic acid). Microscopic evaluation showed one case with poor differentiation of neuroblastoma with $<5 \%$ ganglion cells and other as Gangioneuroblastoma. Immunohistochemistry was positive for neuron specific esterase (NSE), chromogranin A, synaptophysin (Fig. 5e, f, g) and negative for desmin and vimentin.

The rest 2 out of 10 cases were pheochromocytomas, reported in $9 \mathrm{yr}$ male and $30 \mathrm{yr}$ female respectively. Both presented with intermittent pain abdomen associated with giddiness, sweating and headache. Urine VMA was elevated. Microscopy 
showed one with well-formed Zellballen pattern (Fig. 4 ), while other with diffuse solid pattern of tumor cell arrangement. Immunohistochemistry was positive for chromogranin A.

Chart 1: Bar diagram shows incidence of various adrenal lesions

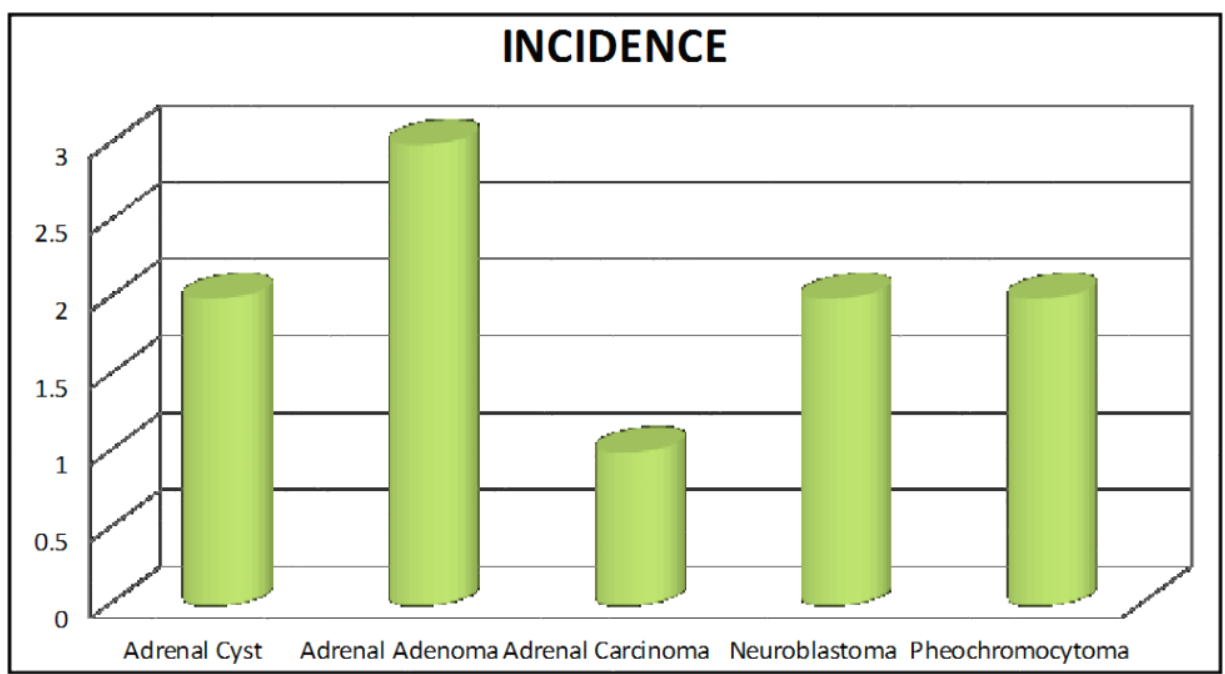

Chart 2: Pie diagram shows female: male ratio in adrenal lesions is $4: 1$
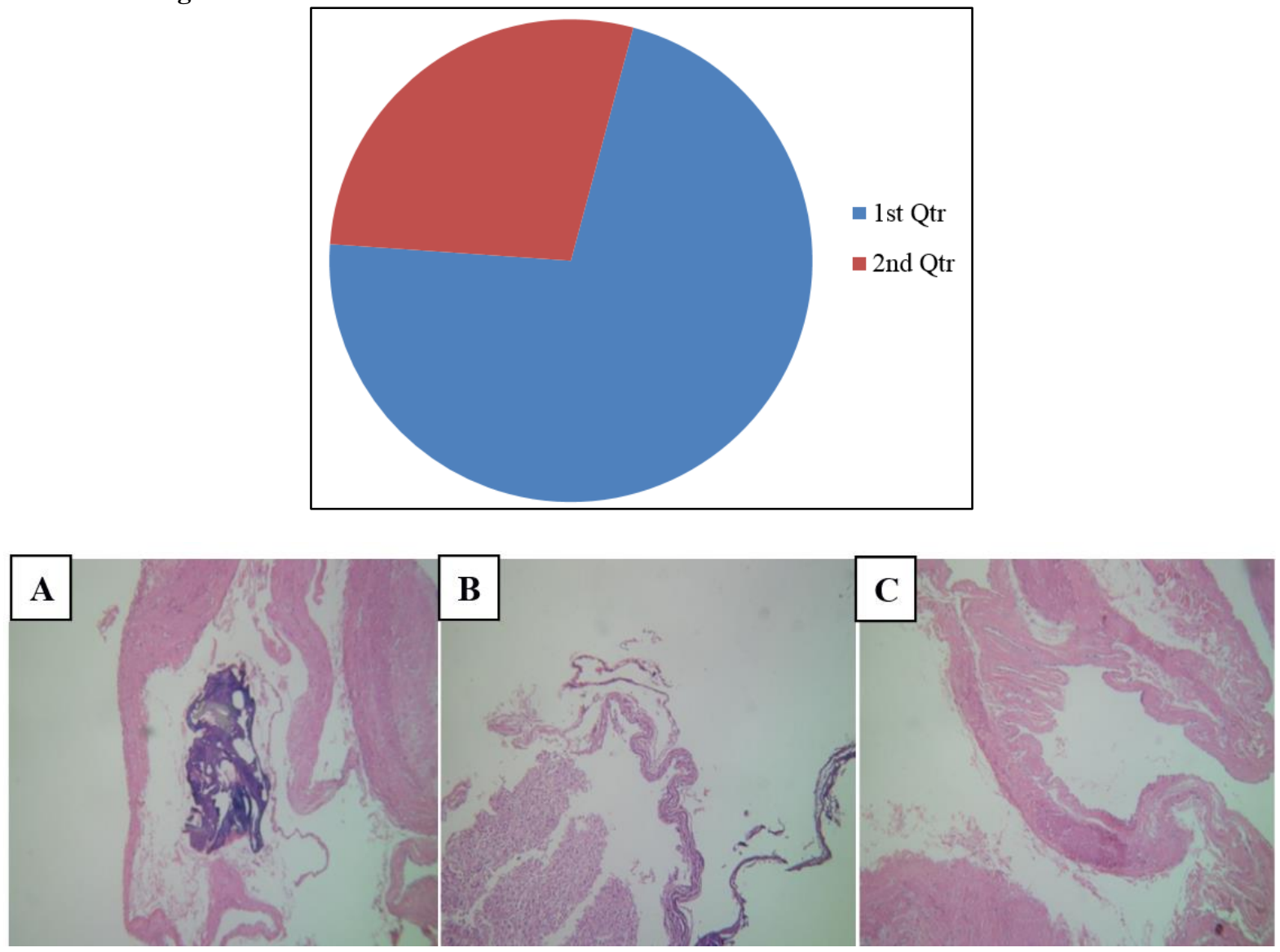

Fig 1: A \& B): Photomicrograph of adrenal cortical cyst -Pseudocyst showing fibrocollagenous wall and calcification (H\& E, 10X); C): Photomicrograph of endothelial cyst showing fibrocollagenous or smooth muscle wall lined by endothelial cells. (H\& E, 10X) 

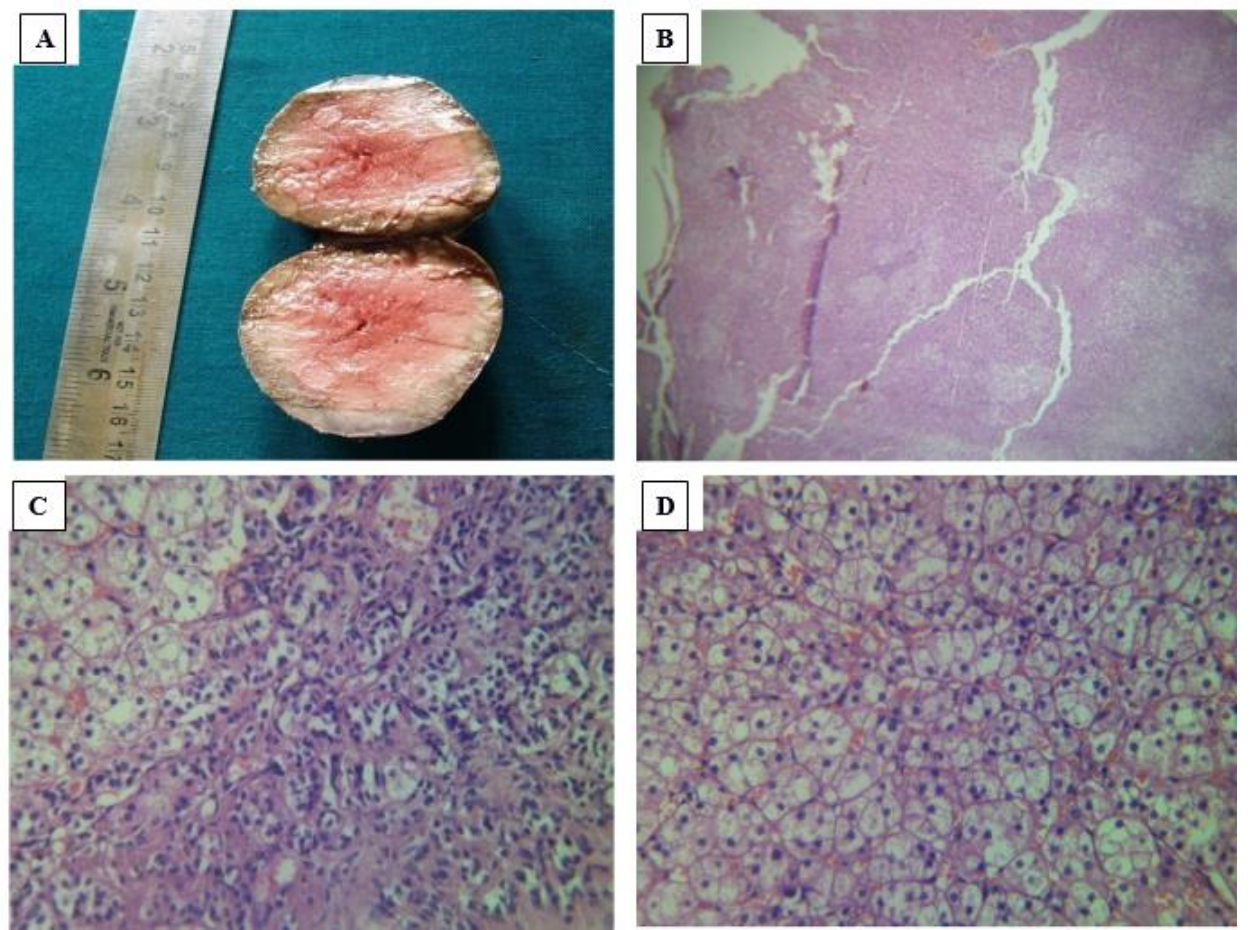

Fig. 2: A): Gross picture of adrenal cortical adenoma after surgery; B): Micrograph of adrenal adenoma (H\&E, 10x) showing capsule; C \& D; Tumor showing round to oval nuclei with small nucleoli, pleomorphic nuclei in some of the cells and few hyperchromatic nuclei $(\mathrm{H \& E}, 40 \mathrm{X})$
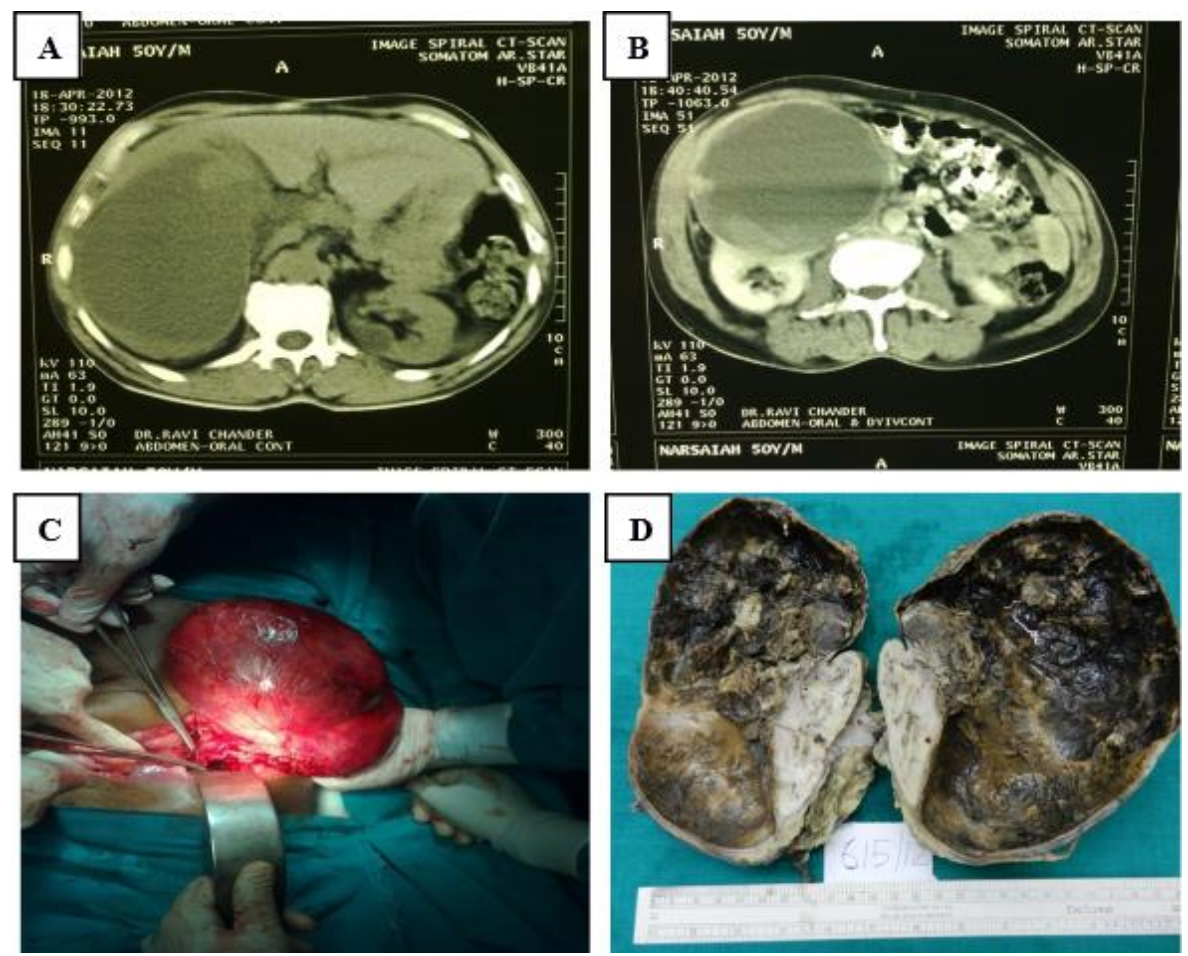

Fig. 3: A): Radiological finding of adrenal cortical carcinoma showing cystic mass arising from the superior pole of right kidney, predominantly cystic \& few solid components at periphery; B): Relationship of mass to kidney predominantly with multiple enhancing solid components; C): Gross picture of adrenal cortical carcinoma after surgery; D): Cut section of the tumor showing size of cyst with kidney msg 19 x 12 x $8 \mathrm{~cm}$ mucin filling with necrotic areas \& haemorrhage 

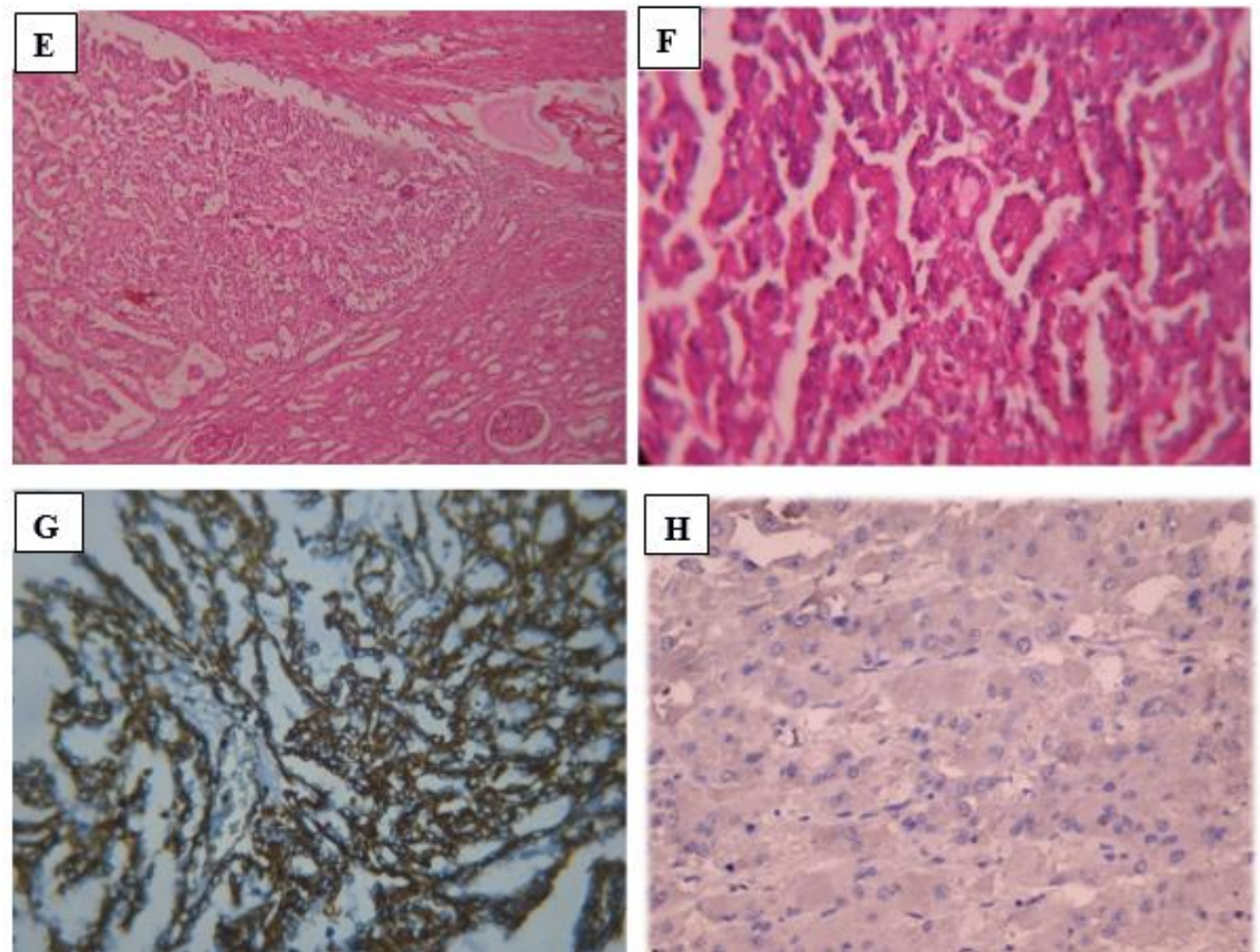

Fig. 3E): Micrograph of adrenal cortical carcinoma shows tumor tissue with pushing margins $(H \& E$, 10x $)$; F): Showing pleomorphic, epithelioid cells with abundant eosinophilic cytoplasm. Trabecular, nested pattern. Intracytoplasmic hyaline globules, multinucleated tumor cells $(\mathrm{H} \& \mathrm{E}, 40 \mathrm{x})$; G): IHC showing positivity; Evimentin; H): Synaptophysin

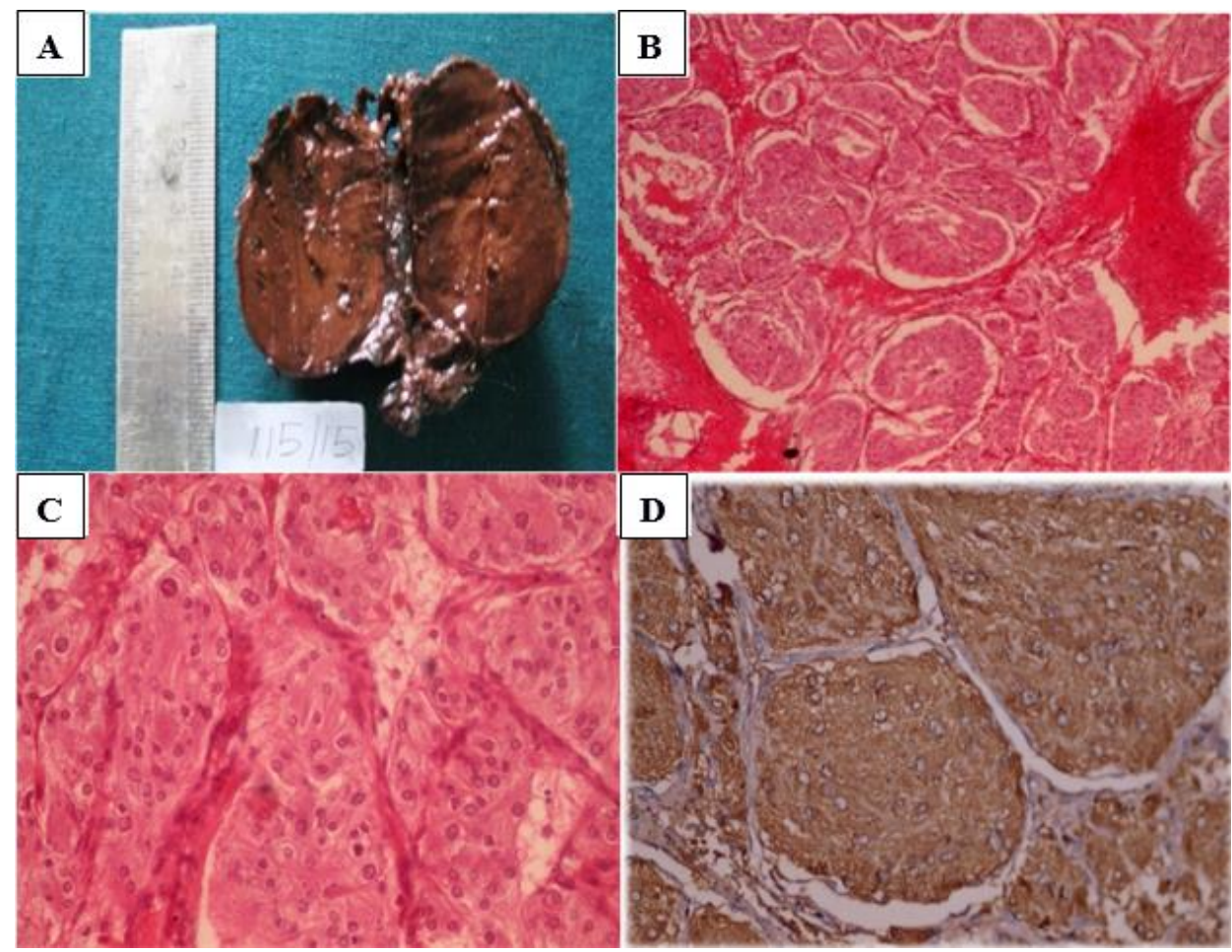

Fig. 4: A): Gross picture of pheochromocytoma after surgery; B) Photomicrograph of pheochromocytoma $(H \& E, 10 x)$ showing large round to polygonal cells with granular eosinophilic cytoplasm with zellballen pattern; C): Pleomorphic hyperchromatic nuclei. (H\&E, 40x); D): IHC showing chromogranin A positivity. 

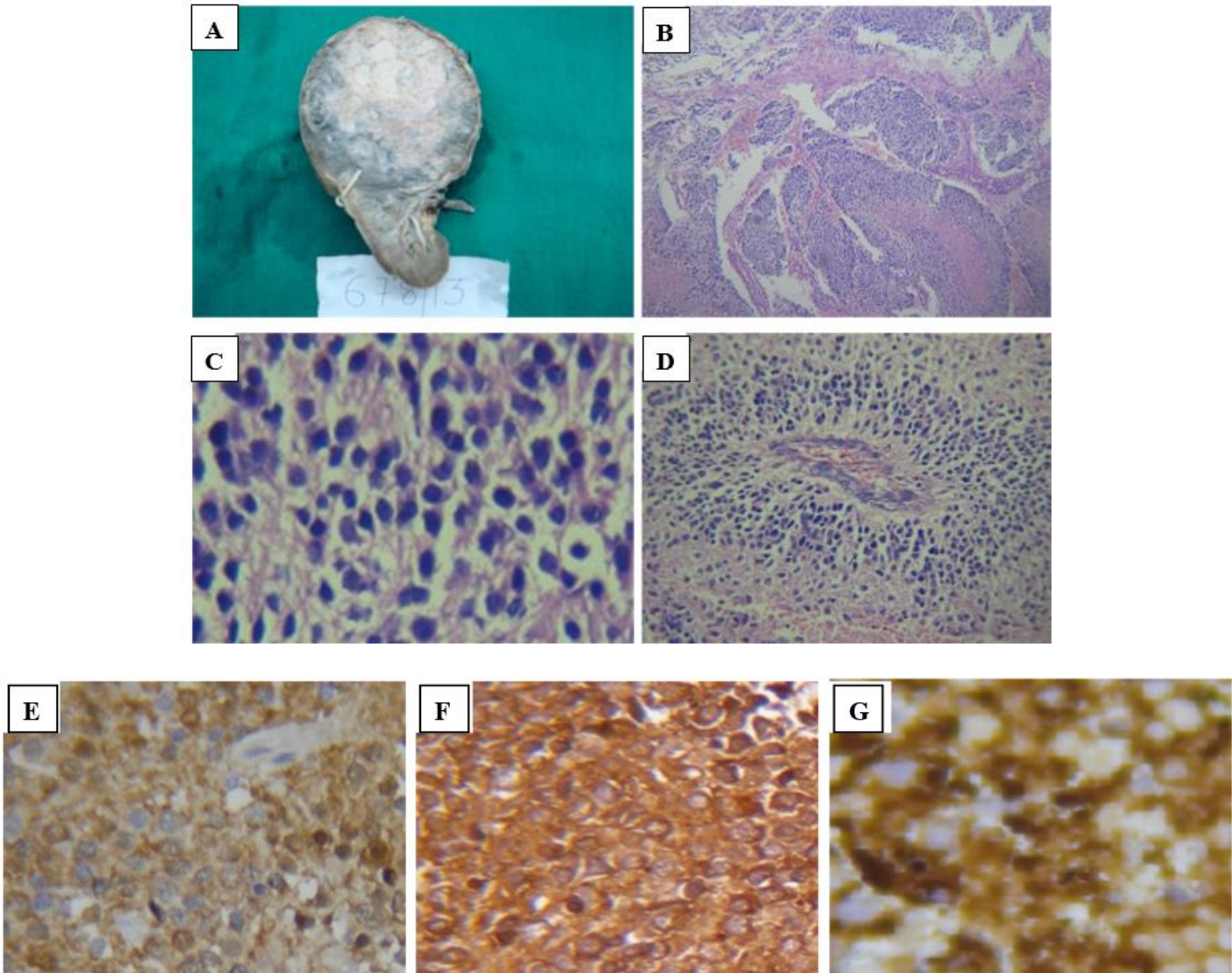

Fig. 5: A): Gross picture of neuroblastoma after surgery; B): Photomicrograph of $(\mathrm{H \& E}, 10 \mathrm{x})$ showing diffusely monotonous small round cells with haemorrhagic and necrotic areas;C\&D): Attempted rosette formation of small round cells around neuropil \& around blood vessels. (H\&E, 40x). E- IHC showing positivity; E): NSE; F): Chromogranin A; G): Synaptophysin

\section{Discussion}

The categorisation and evaluation of adrenocortical neoplasms was always challenging.

Adrenal cysts are non-neoplastic lesions with peak incidence at $5^{\text {th }}$ to $6^{\text {th }}$ decades and female preponderance. Usually patients present with symptoms related to mass effect. Pseudocysts are unilocular cysts containing granulation tissue, brown hemorrhagic fluid and occasionally organizing blood clots with fibrosis (Fig. 1a \& b) and endothelial cyst contents vary from hemorrhagic fluid to fibrin deposition with cleary cyst fluid (Fig. 1c).

Adrenal adenoma are yellow brown tumors and well circumscribed, with round to oval nuclei with small nucleoli, few hyperchromatic pleomorphic nuclei and eosinophilic to vacuolated cytoplasm. (Fig. 2c \& d)

Adrenocortical carcinoma (ACC) is a highly aggressive tumor with a rare annual incidence of 1.5 to 2 per million. It is bimodal in presentation with peaks in pediatric age group and $5^{\text {th }} \& 7^{\text {th }}$ decade. As published in literatures, our case presented symptoms related to mass effect. The functional tumors may show cushings syndrome, virilisation, ${ }^{14}$ feminization and hyper aldosteronism. The Weiss criteria is used to grade tumor as malignant. The IHC of tumor showed positivity for vimentin and synaptophysin and negative for cytokeratin supporting the diagnosis.

Neuroblastoma characterised by neuroblasts and epithelial-like cells ${ }^{15}$ with calcification and cystic changes, ganglion cells, Schwann cells and Mitotic karyotic index (MKC) used to classify as: Low $-<2 \%$ of MKC, Intermediate - 2\% - 4\% of MKC, High - >4\% of MKC.NSE, chromogranin, synaptophysin done.

Pheochromocytomas: Adrenal medullary neuroendocrine tumor with incidence of 2-8 per million and peaks at $4^{\text {th }}-5^{\text {th }}$ decade. The classic triad includes Paroxysmal headache, diaphoresis, tachycardia. They secrete excess catecholamines which may cause hypertensive crisis, cardiovascular stoke, MI or multiple organ failure. In our study we had pediatric case with complaints of headache, vomiting and raised blood pressure which is similar to others finding.

The study by Stewart et al (2004) which included 9 cases of adrenocortical tumors showed: The mean age 
of presentation as 2 Year and 5 months, male to female ratio as 1.3:1 and the common presentation as abdominal distention. Particularly virilisation was mostly associated with adrenocortical carcinoma and cushingoid features with adrenal adenoma. (Table 2).

The study by Chen qui Li et al (2008) which included 73 cases of adrenal medullary tumors over a duration of 20 years showed: The peak age of presentation was 2-5 years, Male to female ration as 1.8:1 and the common presentation as Mass per abdomen (Table 3) and our present study is in concordant with this study.

Table 1: Presenting symptoms of patients

\begin{tabular}{|l|c|}
\hline \multicolumn{1}{|c|}{ Symptoms } & Number of Cases \\
\hline Mass Abdomen & 10 \\
\hline Pain Abdomen & 6 \\
\hline Cushing syndrome & 2 \\
\hline Virilization & 1 \\
\hline Giddiness, Sweating & 1 \\
\hline
\end{tabular}

Table 2: The following table shows comparision between present study and Stewart et al (2004)

\begin{tabular}{|l|c|c|}
\hline & Stewart et al (2004) & Present study \\
\hline No. of cases & 09 & 6 \\
\hline Duration of study & 29 years & 5 years \\
\hline M:F & $5: 4$ & $2: 1$ \\
\hline Chief complaint & Abdomen distension & Abdomen distension \\
\hline Cushing syndrome & 8 cases & 2 cases \\
\hline Virilisation & 4 cases & 1 case \\
\hline Adenoma: carcinoma & $1: 8$ & $2: 1$ \\
\hline
\end{tabular}

Table 3: The following table shows comparision between present study and Chen qui li et al (2004)

\begin{tabular}{|l|c|c|}
\hline & Chen qiu li et al & Present study \\
\hline No. of cases & 73 & 4 \\
\hline Duration of study & 20 years & 05 years \\
\hline M:F & $1.8: 1$ & $1: 0$ \\
\hline Chief presenting complaint & Mass abdomen & Mass abdomen \\
\hline Peak age of onset & $2-5$ years & $0-1$ year \\
\hline
\end{tabular}

\section{Conclusion}

It is always challenging task for pathologists to distinguish between benign and malignant adrenal lesions. So, even though clinical, radiological, biochemical evaluations have their parts, microscopic evaluation and Immunohistochemistry helps in identifying the tumors up to cellular level, categorize them properly and thus helping in proper diagnosis and management of adrenal tumors.

\section{References}

1. E. Carvounis. Vascular adrenal cysts a brief review of the literature. Arch Pathol Lab Med 2006;130:1722-1724.

2. W. Saegar. Histopathological classification of adrenal tumors. European journal on clinical investigation 2003;30(3):58-62.

3. Romolo Sandrini. Childhood adrenocortical tumors. The Journal of Clinical Endocrinology \& Metabolism. 1997;82(7):2027-2031.

4. Hough AJ, Hollified JW, Page DL, Hartmann WH. Prognostic factors in adrenal cortical tumors. A mathematical analysis of clinical and morphologic data. Am J Clin Pathol. 1979;72:390-399.

5. Van Slooten H, Schaberg A, Smeenk D, Moolenar AJ. Morphologic characteristics of benign and malignant adrenocortical tumors. Cancer. 1985;55:766-773.

6. Pacak K, Linehan WM, Eisenhofer G. Recent advances in genetics, diagnosis, localization and treatment of pheochromocytoma. Ann Intern Med 2001;134:315-320.
7. Weiss L. Comparative histologic study of 43 metastasizing and non-metastasizing adrenocortical tumors. Am J Surg Pathol 1984;8:163-169.

8. Weiss LM, Medeiros LJ and Vickery AL. Pathologic features of prognostic significance in adrenocortical carcinoma. Am J Surg Pathol 1988;13:202-206.

9. A Blanes. Histologic Criteria for Adrenocortical Proliferative Lesions. Am J Clin Pathol 2007;127:398408.

10. Jain M, Kapoor S, Mishra A, Gupta S, Agarwal A. Weiss criteria in large Adrenocortical tumors: a validation study. Indian journal pathol and microbiol 2010;53(2):222-226.

11. Sroujreh A.S, Farah G.R, Haddad M.J. Adrenal cyst: diagnosis and treatment. Br J Urol, 1990;65:570-575.

12. A. Wedmid, M. Palese. Diagnosis and treatment of the adrenal cyst. Curr Urol Rep, 2010;11:44-50.

13. Sasano H. Recent advances in histopathology and immunohistochemistry of adrenocortical carcinoma. Endocrpathology 2006;17(4):345-54.

14. Kenny FM, Hashida Y, Askari A. Virilizing tumors of the adrenal cortex. Am J Dis Child 1968;115:445-448.

15. Chen Qiu Li. Clinical Analysis of Adrenal Neuroblastoma in 73 Children. Journal of Applied clinical pediatrics 2008;20:140-48.

How to cite this article: Karre S, Gorva A, Shanmugam C, Gorrela VDPK, Veeragandham S. Histopathologic spectrum of adrenal lesions. Ind J Pathol Oncol, 2018;5(3):463-469. 